\title{
Measurement of permeability using a bench-top centrifuge
}

\author{
C. ANDERSON*, V. SIVAKUMAR $\dagger$ and J. A. BLACK
}

\begin{abstract}
The commonly used British Standard constant head triaxial permeability test for testing of finegrained soils is relatively time consuming. A reduction in the required time for soil permeability testing would provide potential cost savings to the construction industry, particularly in the construction quality assurance of landfill clay liners. The purpose of this paper is to evaluate an alternative approach of measuring permeability of fine-grained soils benefiting from accelerated time scaling for seepage flow when testing specimens in elevated gravity conditions provided by a centrifuge. As part of the investigation, an apparatus was designed and produced to measure water flow through soil samples under conditions of elevated gravitational acceleration using a small desktop laboratory centrifuge. A membrane was used to hydrostatically confine the test sample. A miniature data acquisition system was designed and incorporated in the apparatus to monitor and record changes in head and flow throughout the tests. Under enhanced gravity in the centrifuge, the flow through the sample was under 'variable head' conditions as opposed to 'constant head' conditions as in the classic constant head permeability tests conducted at $1 \mathrm{~g}$. A mathematical model was developed for analysis of Darcy's coefficient of permeability under conditions of elevated gravitational acceleration and verified using the results obtained. The test data compare well with the results on analogous samples obtained using the classical British Standard constant head permeability tests.
\end{abstract}

KEYWORDS: centrifuge modelling; clays; groundwater; landfills; permeability

\section{INTRODUCTION}

Low-permeability soil barriers are often used in geotechnical works for controlling leakage flow and contaminant migration. There are several methods of measuring the permeability of soils including: the British Standard (BS) flexible boundary constant head permeability (BS 1377, Part 6:1990, BSI (1990)); the accelerated permeability (AP) test (Environment Agency, 2003); the ramped accelerated permeability (RAP) test; and several in-situ measurement techniques (Olsen et al., 1985; Aiban \& Znidarcic, 1989; Huang et al., 1998a, 1998b). In the case of landfill applications, as part of construction quality control (CQA) to ensure that liner materials have sufficiently low permeability, tests are carried out on undisturbed samples recovered from the site or representative samples produced in the laboratory. Within the UK, regulations specify that permeability should be measured in accordance with BS 1377: Part 6:1990 (BSI, 1990), method 6. The BS test is a relatively time-consuming procedure with turnaround time up to 2 months from time of sampling to report of results. It is shown (by two of the present authors and colleagues in a paper that is under review) that the AP method does not yield any significant improvement in the time saving, while the permeability values obtained using AP are up to ten times lower than those derived using the BS test. The purpose of this paper is to investigate the use of a laboratory-based centrifuge to measure the permeability of fine materials and to examine whether the results are comparable with measurements obtained using the BS procedure.

Centrifuge modelling has been long recognised as a tool which, by elevation of the force of gravity, can achieve similarity of stresses between small-scale models and full-

Manuscript received 4 July 2013; revised manuscript accepted 2 December 2014

Discussion on this paper is welcomed by the editor.

* Byrne Looby Partners, Belfast, UK.

$\uparrow$ Queen's University Belfast, Belfast, UK.

$\$$ University of Sheffield, Sheffield, UK. scale prototypes under in-situ conditions (Schofield, 1980; Garnier et al., 2007). Centrifuges are normally used to model prototype situations to provide an independent evaluation of performance for many geotechnical problems, for example, mining (Bucky, 1931), retaining structures (Bolton \& Powrie, 1988), embankments (Take \& Bolton, 2011) and offshore foundations White (2008). They can also be used to measure material characteristics such as the diffusion coefficient, retardation factor and the coefficient of permeability. A centrifuge accelerates water flow in porous soils and therefore it can reduce the time for testing low-permeability soils compared to conventional procedures. Some centrifuge set-ups can also accommodate up to six samples, allowing multiple tests to be run simultaneously (Mitchell, 1998).

Mitchell (1993, 1994a, 1994b, 1998) and Theriault \& Mitchell (1997) carried out permeability tests using a small centrifuge and, in their testing procedure, the flow of permeant into and out of the sample was measured once the centrifuge had stopped. Singh \& Gupta $(2000,2001)$ adopted a procedure in which the flow was measured in-flight by observing the permeant level through a small window on a $0.63 \mathrm{~m}$ rotor centrifuge using a stroboscope. In their studies the outflow head was maintained constant. Singh \& Gupta (2001, 2002) demonstrated the reliability of permeability values obtained using this set-up when compared with standard procedures (i.e. the BS test). The present paper presents experimental observations and interpretation of data for calculating the coefficient of permeability of fine soils in which an in-flight automated system is used to measure the inflow and outflow of water through the soil in a bench-top laboratory centrifuge. The term 'permeability' is interchangeable with 'coefficient of permeability' or 'Darcy's permeability'. The latter term is generally used throughout the paper.

\section{USE OF SCALING FACTORS TO DEFINE DARCY'S COEFFICIENT OF PERMEABILITY}

The scaling effect is significant in the research reported here; therefore, a brief summary of this aspect is discussed 
herein. As with all disciplines in which physical models are used, in geotechnical centrifuge modelling and testing there is a tendency to use scaling factors appropriate to the models and prototypes under investigation. Muir-Wood (2004) states that, provided the height of the model is less than approximately $0 \cdot 1$ times the radius of rotation, the variation of the acceleration field along the sample is generally considered marginal and can be assumed constant. However, if the length of the sample is more than $0 \cdot 1$ times the radius of rotation then the acceleration along the sample length, and therefore the acceleration ratio $N$ (ratio between the acceleration in the centrifuge at a given point and the earth's gravity $\boldsymbol{g}$ ), is variable and thus should not be considered constant. This situation generally prevails in small geotechnical laboratory centrifuges (Black, 2014).

In order to formulate an appropriate analysis for centrifuge permeability testing, consideration must first be given to the scaling laws applied to seepage flow. From the literature (Garnier et al., 2007) relating to the scaling laws for centrifuge modelling it is well established that seepage velocity, $v$, is scaled directly by the factor $N$, where

$$
v_{\mathrm{m}}=N v_{\mathrm{p}}
$$

where $v_{\mathrm{m}}$ is the model seepage velocity and $v_{\mathrm{p}}$ is the seepage velocity in prototype. This scaling relationship has been verified by a number of authors studying both steadystate and transient seepage flow in centrifuge modelling (Cargill \& Ko, 1983; Arulanandan et al., 1988; Khalifa et al., 2000; Singh \& Gupta, 2000). There is, however, controversy within the literature regarding whether it is the permeability, $k$, or the hydraulic gradient, $i$, that is a function of $N$. This controversy has been raised by various authors including Goodings (1979, 1985), Butterfield (2000), Dean (2001) and Madabhushi \& Thusyanthan (2003). However, it is commonly overlooked as the seepage velocity is generally the value under investigation, and irrespective of whether $k$ or $i$ is the scaled parameter, the end result, $v_{\mathrm{m}}=N v_{\mathrm{p}}$, remains the same. This method may be satisfactory where it is appropriate to consider $N$ as a constant throughout the depth of a model; however, if there is a requirement for the consideration of $N$ as a variable related to radius of rotation (as in the present investigation discussed later), then a more thorough understanding of the parameters to be scaled is necessary.

Cargill \& Ko (1983), Tan \& Scott (1985), Mitchell (1998) and Singh \& Gupta (2000) defined the hydraulic gradient $i$ as independent of gravity and considered permeability $k$ to be directly proportional to gravity with a resultant scaling factor of $N$. The formal definition of hydraulic gradient, as presented in the majority of the literature, is the ratio of change in total head, $\Delta h$, and the length, $L$, over which that change in head occurs. When scaling of dimensions of a centrifuge model both $L$ and $\Delta h$ will be $N$ times smaller in the model, and as a result the hydraulic gradient can be argued to be the same in the model and prototype (i.e. $\left.i_{\mathrm{m}}=i_{\mathrm{p}}\right)$.

The parameter known as the intrinsic permeability $\kappa$, derived by Muskat (1937), is shown in equation (2) related to Darcy's coefficient of permeability $k$ by the following expression

$$
k=\kappa \frac{\gamma}{\mu}=\kappa \frac{\rho g}{\mu}
$$

where $\gamma, \mu$ and $\rho$ are the unit weight, dynamic viscosity and the density of the fluid, respectively. This shows that because Darcy's coefficient of permeability is directly proportional to the unit weight of the permeant $\gamma$ then under conditions of elevated gravity $k$ will increase $N$ times (i.e. $k_{\mathrm{m}}=N k_{\mathrm{p}}$ ) on the assumption that the dynamic viscosity is unaffected by gravity.

Alternatively, Schofield (1980), Goodings (1985) and Taylor (1987) have all suggested permeability to be independent of gravity and that the hydraulic gradient has the scaling factor of $N$. Taylor (1987) highlights an inconsistency with regard to the relationship shown in equation (2), due to the direct relationship between permeability and gravity equation (2) suggests that any given soil mass in space at zero gravity is impermeable. However, this is not the case, as a soil mass has a given amount of pore space irrespective of gravity and, if a fluid with an applied pressure gradient is present across the boundary of that soil mass, then permeant flow will take place. Goodings (1985) suggests that Darcy's coefficient of permeability, $k$, should not be scaled and should be considered as a material constant.

Madabhushi \& Thusyanthan (2003) present an argument for the scaling of hydraulic gradient using the illustration shown in Fig. 1. If the model sample is rotated in a centrifuge applying a gravitational acceleration of $N \boldsymbol{g}$ then, assuming that the radius of rotation is large enough to disregard large variations in $N$, the soil model can represent a prototype soil length of $N \times L$. The static pressure difference between points ' $\mathrm{B}$ ' and ' $\mathrm{C}$ ' for the centrifuge model is $L \rho(N \boldsymbol{g})$, and for the prototype soil is $(N L) \rho \boldsymbol{g}$. Similarly, the potential energy across the sample is the same for both model and prototype if the inlet and outlet permeant heads are held constant at the points 'A' and 'D', respectively. Madabhushi \& Thusyanthan (2003) point out that, because the driving force on permeant flow is the same for both model and prototype and the flow length is $N$ times shorter in the model than in the prototype, an argument for scaling hydraulic gradient may be made (i.e. $i_{\mathrm{p}}=N i_{\mathrm{m}}$ ).

The cause of the confusion in scaling parameters for permeant flow under accelerated gravity is due to the conventional definitions of hydraulic gradient $i$ and Darcy's permeability $k$. It should be noted that Darcy's equation for permeant flow is an empirical equation, with the conventional definition of the hydraulic gradient $i$ representing the driving force for flow, but without the consideration of gravitational acceleration $\boldsymbol{g}$. Instead acceleration due to gravity is considered within Darcy's coefficient of permeability $k$ as the unit weight of the fluid (as shown in equation (2)). This makes Darcy's coefficient of permeability, $k$,

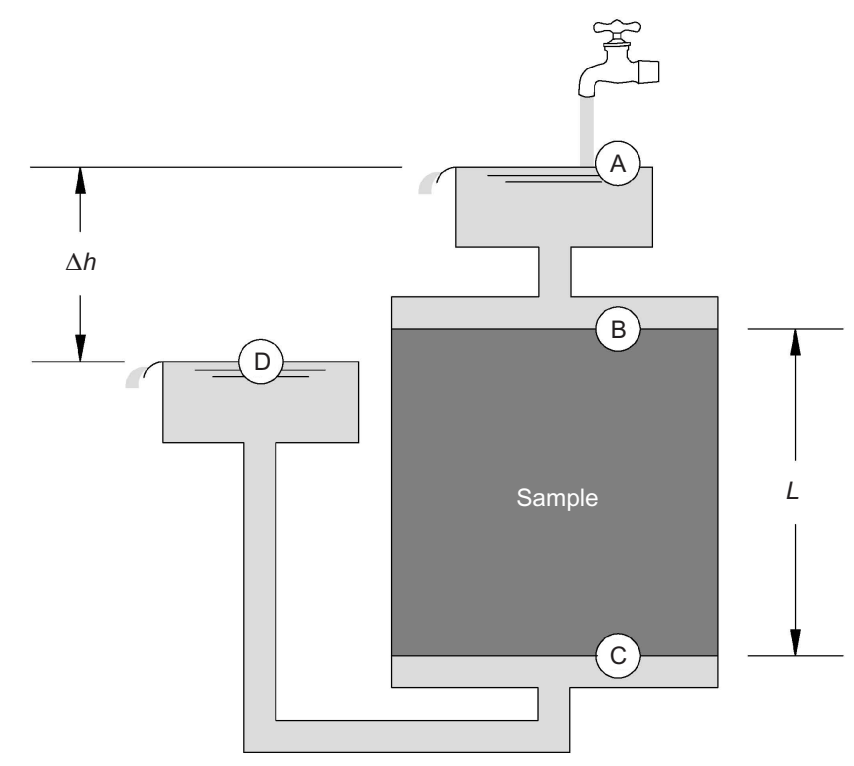

Fig. 1. Free-body diagram of water under centrifuge action (Madabhushi \& Thusyanthan, 2003) 
directly proportional to gravity, and relevant under conditions of normal gravitational acceleration (i.e. $N=1$ ). For this reason, and giving consideration to the variability of gravitational acceleration experienced within a small centrifuge, it is erroneous to use a scaling relationship, to define a value for $k$, when using a small centrifuge permeameter. Instead, Madabhushi \& Thusyanthan (2003) suggest that for centrifuge modelling (or assessing permeant flow in a centrifuge), gravity should be represented by the energy gradient (potential energy gradient + pressure energy gradient) which drives the permeant flow.

From Bernoulli's principle, the energy required for permeation is the energy associated with the movement of the permeant (velocity head), plus the energy from the pressure in the permeant (pressure head), plus the energy from the height of the fluid relative to an arbitrary datum (elevation head); such that

$$
\Delta h=\left(\frac{\Delta v^{2}}{2 \boldsymbol{g}}\right)+\left(\frac{\Delta u}{\rho \boldsymbol{g}}\right)+\Delta z
$$

where $u, v$ and $z$ are pore-water pressure, velocity of the water flow and elevation above datum, respectively. Lambe \& Whitman (1979) state that, for fluid flow through soils, velocity head is too small to be of any consequence and may therefore be neglected. With consideration of equations (2) and (3), seepage velocity may be represented by the following relationship

$$
v=\kappa \frac{\rho \boldsymbol{g}}{\mu} \times \frac{[(\Delta u / \rho g)+\Delta z]}{L}
$$

where $\Delta u$ and $\Delta z$ are the change in permeant pressure and the change in elevation over a distance $L$, parallel to the direction of fluid flow across the permeated medium, respectively. Rearranging equation (4), an expression which relates the energy gradient per unit volume of fluid as the driving force for flow (i.e. energy difference) may be derived. Unlike hydraulic gradient the energy is directly proportional to gravitational acceleration

$$
v=\frac{\kappa}{\mu} \times \frac{\Delta(u+z \boldsymbol{g} \rho)}{L}
$$

As the intrinsic permeability relationship derived by Muskat (1937) is regarded as valid under conditions of normal gravity then

$$
k_{1 g}=\kappa \frac{\gamma_{1 g}}{\mu}
$$

A relationship between Darcy's coefficient of permeability and the energy gradient per unit volume of fluid may then be defined as

$$
v=\frac{k_{1 g}}{\gamma_{1 g}} \times \frac{\Delta(u+z \boldsymbol{g} \rho)}{L}
$$

where $k_{1 g}$ and $\gamma_{1 g}$ are Darcy's coefficient of permeability at $1 \boldsymbol{g}$ and the unit weight of the permeant at $1 \boldsymbol{g}$, respectively. The use of equation (7) allows the interpretation of the coefficient of permeability under conditions of elevated gravitational acceleration and is fundamental to the accurate analysis of centrifuge permeability tests.

\section{EXPERIMENTAL PROGRAMME AND ANALYTICAL}

MODEL

General arrangement and overview

The investigations were carried out using a Sigma 235 bench-top centrifuge which has a $0.20 \mathrm{~m}$ rotor and four 0.751 containers to locate the proposed permeameter set-up. The specific design requirements were

(a) the sample size should be small enough to be accommodated within the centrifuge cups of the centrifuge

(b) the device should be lightweight and it should include chambers to hold reservoirs of sufficient quantities of permeant for the inflow and outflow volume

(c) it should provide automated measurement of permeant inflow and outflow and an estimation of the hydraulic gradient/pressure differential applied across the sample during testing (i.e. in-flight data recovery)

(d) flexible lateral boundary conditions should be achieved

(e) there should be automated opening of the inlet valve only when sufficient lateral pressures are applied to the sample, to negate preferential flow between the sample sides and the flexible lateral boundary.

Figure 2 shows the general arrangement of the prototype apparatus, which can accommodate cylindrical soil samples $75 \mathrm{~mm}$ in diameter and $30 \mathrm{~mm}$ long. The sample is axially confined between the bottom and top caps (which include porous stones) and laterally confined by a $75 \mathrm{~mm}$ diameter rubber membrane. A drainage line is provided in the bottom cap to facilitate the inflow of permeant using a flexible drainage line connected by way of a spring gravity valve from the inlet chamber. A similar arrangement is provided for the outflow from the top of the sample to the outlet chamber. The inlet and outlet chambers consist of two concentric cylinders, permanently connected to a circular base. These chambers are suspended above the sample by a hanging bracket supported by the top of the centrifuge cup. In order to provide a flow through the sample, the inlet chamber is filled with permeant to a higher level than that in the outlet chamber, thereby producing a pore-water pressure differential gradient across the sample. Under elevated gravity, this gradient increases, producing an increased flow of permeant from the inlet chamber upwards through the sample, and into the outlet chamber. As such, the flow condition applied during the test is essentially a falling head/rising tail condition. Ignoring the effects of head loss through the system, the flow would continue until the permeant levels in the chambers equalise.

Miniature pressure transducers incorporated into the base of both inlet and outlet chambers facilitate the measurement of pressure head generated from the volume of water retained in each chamber under elevated gravity during centrifuge testing. Using the pressure obtained from each pressure cell, and knowing the rotational speed of the centrifuge, a mathematical model derived for this device (discussed later) allows calculation of changes in inlet and outlet volumes during testing, and calculation of water pressures applied to the sample base and the top of the sample. As a result, pressure changes monitored by the miniature pressure cells may be directly correlated to the permeability of the sample. Both pressure transducers are connected to a data logger (by way of plug-in sockets, Fig. 3), which is secured to the top of the permeant chambers, after permeant levels within the inner and outer chamber have been filled as necessary during setup. The centrifuge permeameter is set to take readings at specific intervals during the test using a programmable microprocessor.

A membrane was used to hydrostatically confine the test sample. As previously detailed, during centrifuge tests, vertical stress on the sample will increase due to the increasing 


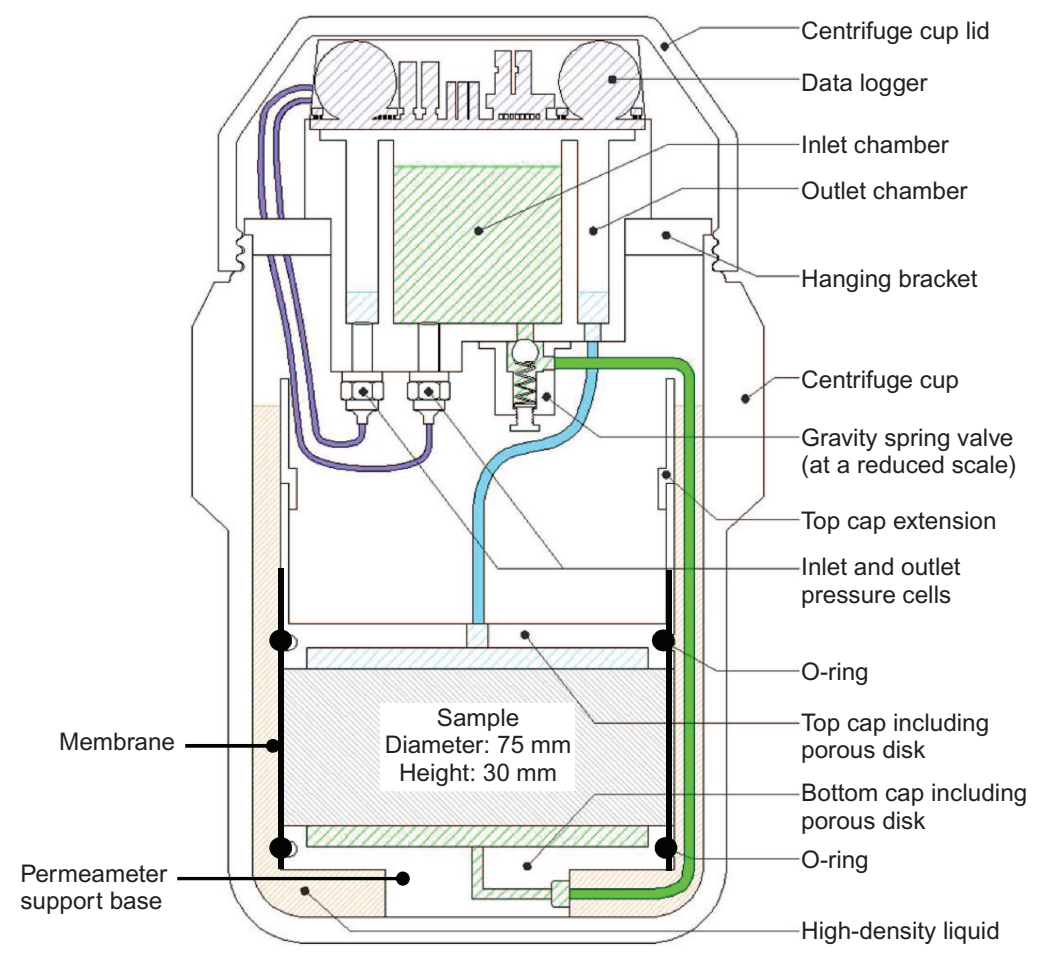

(a)

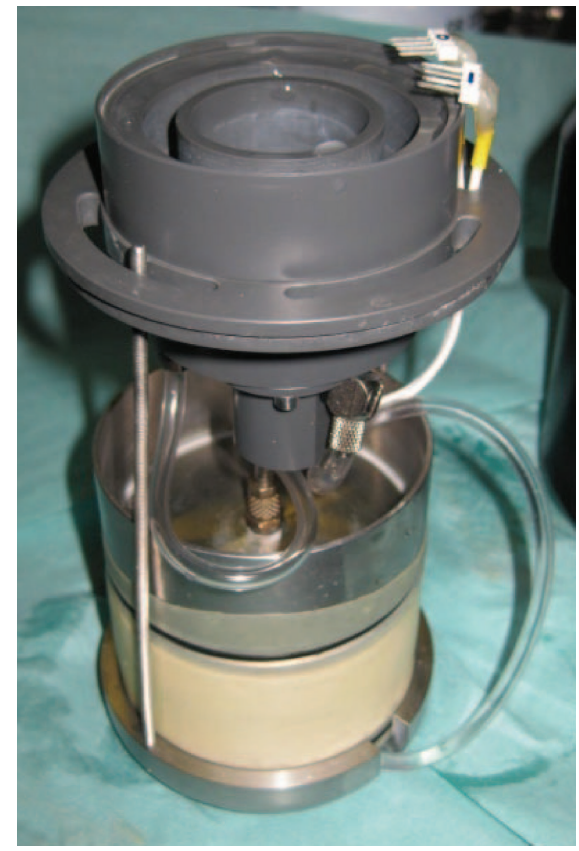

(b)

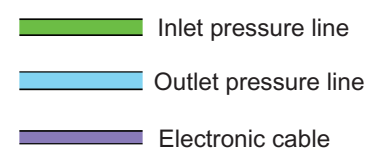

Note: To provide clarity within this schematic some components of the apparatus have been omitted, including details of the fixtures and fittings, the permeameter base support and the confining membrane. All detailed components are drawn to scale with the exception of the gravity spring valve, which has been reduced to allow clarification of flow paths.

Fig. 2. Centrifuge permeability system: (a) schematic diagram; (b) photograph

acceleration, both from its own self-weight and the applied load from any mass resting on top of the sample. This in turn could lead to lateral straining, and in extreme cases, shearing of the sample, which may affect the permeant flow and therefore yield unrepresentative permeability results. In order to minimise loading of the sample, the inlet and outlet chambers and data-logging component are suspended using the hanging bracket shown in Fig. 2 and the permeant line connections between the chambers and the sample are highly flexible. In order to ensure minimal lateral straining of the sample during centrifuge, the vertical stresses imposed by the top cap and the sample self-weight are balanced using lateral confining pressures surrounding the flexible membrane around the sample. This confining pressure is generated using a high-density liquid (HDL), corresponding to the bulk density of the soil sample, positioned inside the centrifuge cup laterally surrounding the sample, top cap and bottom cap. As the material used for the production of the top caps has a higher density than the sample or HDL, to allow equalisation of vertical and horizontal pressures at the sample top, the top cap was designed with elongated walled sides to allow an appropriate increased head of HDL to be used.

Under flexible boundary conditions, a requirement to ensure no preferential flow between the membrane and the sample sides is a minimum effective confining pressure of $15 \mathrm{kPa}$ (Mitchell, 1994b). The maximum permeant pressure applied to the sample is defined by the permeant head in the inlet chamber at the start of the test. As the confining pressure generated by the HDL increases laterally from the top to the base of the sample, to account for the required effective confining pressures and minimise the divergence in effective pressure across the sample length, the inlet flow is applied to the base of the sample. Hence an upward flow is generated through the sample during testing. The gravitycontrol valve was used to prevent permeant flow from the inlet chamber until the rotational speed in the centrifuge was sufficient to generate an effective confining pressure of $15 \mathrm{kPa}$ to negate preferential flow.

The electronic component associated with the centrifuge permeameter is relatively simple in design and operation. The reliability of the data logger under elevated acceleration was examined in previous research (Brown et al., 2009). The data logger consists of off-the-shelf components, with the exception of the printed circuit board (PCB), which was designed and manufactured at the Queen's University Belfast (QUB) workshop. The major restriction for the selection of electrical components was their size, given the relatively small space in which the data-logging component was to be housed (Fig. 2).

The electronic data logger is pictured in Fig. 3. The two pressure cells are connected to separate amplifiers, which amplify the voltage output from the pressure cells. The amplified signal from both load cells is fed into an analogueto-digital converter (ADC). This digital signal is then fed to a microprocessor, known as a 'Basic' stamp. It is essentially an extremely small printed circuit board, which contains the essential elements of a microprocessor system, including: a microcontroller containing the central processing unit (CPU), a built-in read-only memory (ROM) containing the Basic (i.e. programming language) interpreter. In addition to the storage of data, the stamp performs a number of other roles, and essentially controls the workings of the data-logging unit. Two $3.6 \mathrm{~V}$ lithium-ion batteries supply a $7 \cdot 2 \mathrm{~V}$ power supply directly to the stamp. the stamp contains a regulator, and therefore redistributes a regulated $5 \mathrm{~V}$ power supply to the remaining circuit (i.e. the two load cells, two amplifiers 


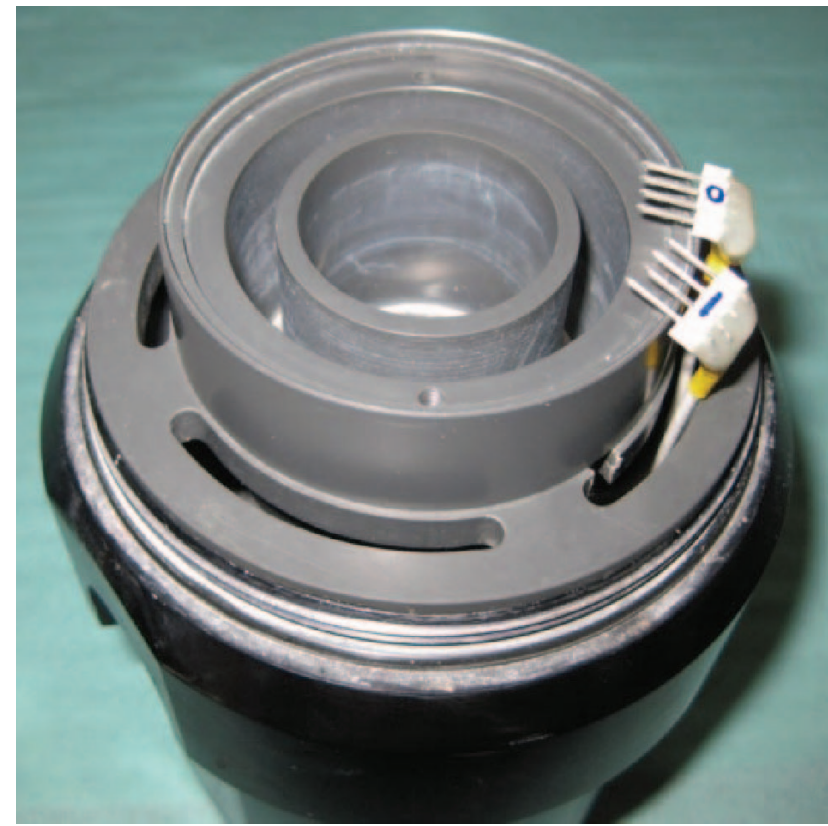

(a)

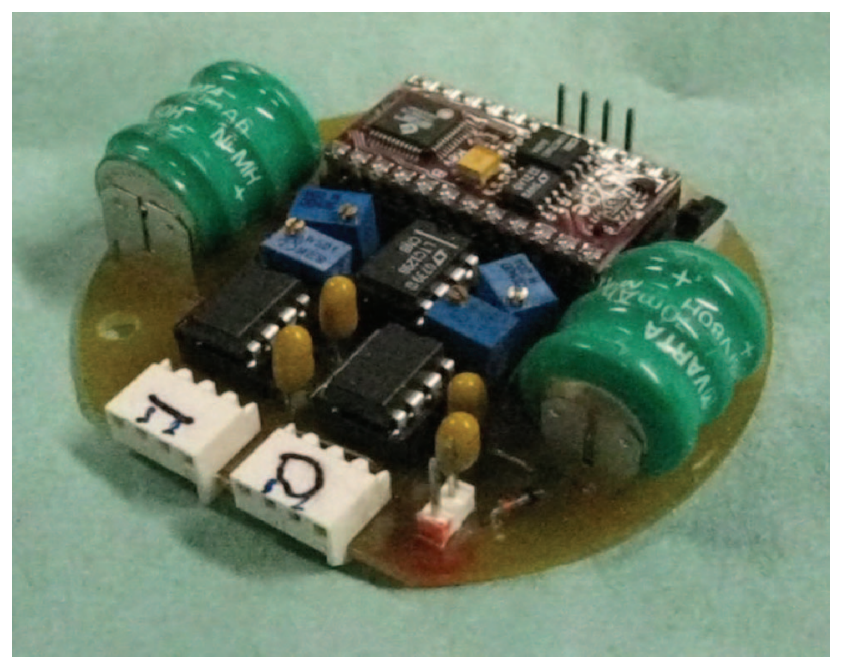

(b)

Fig. 3. (a) Water reservoir for inlet and outlet; (b) data logger

and the $\mathrm{ADC}$ ). It was found that continuous power supply to these other components led to a greatly shortened battery life, which was incompatible with the required test duration. In order to address this, the software written for the stamp was modified to control the power supply to these additional components, which are now 'powered up' and 'powered down' immediately before and after a reading is taken. Each pressure cell was routinely calibrated using a desk-top pressure calibrator, both periodically and after any adjustment was made to the circuit. Brown et al. (2009) confirmed that the calibration factor was not affected by acceleration up to $600 \mathrm{~g}$.

In order to check the above-mentioned calibration, the apparatus (excluding the sample, caps and HDL) was tested in the centrifuge, both periodically and after any adjustment was made to the circuit. Known quantities of water, measured to the nearest $0.01 \mathrm{~cm}^{3}$, were placed in both the inlet and outlet chambers and the centrifuge was run at various accelerated gravities. The bases of both chambers were sealed and therefore there was no flow through the inlet or outlet tubing. Fig. 4 shows the raw data obtained from the calibration test. The key details the amount of water in the

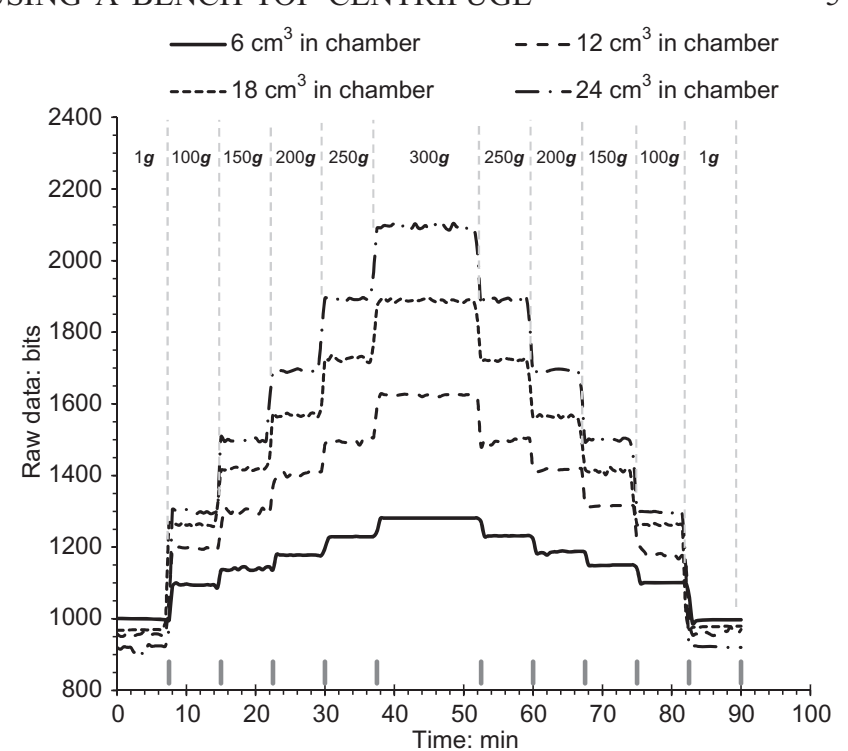

Fig. 4. Pressure cell output plotted against time (and the influence of gravitational acceleration)

chamber for each plot, while the $\boldsymbol{g}$ values presented correlate to the $N$ value applied in the centrifuge.

\section{Mathematical model}

The analysis is based on an Excel spreadsheet, which allows the user to input testing variables and visualise the resultant stresses imposed on the sample, taking into account the variation in the gravitational acceleration with distance from the point of rotation. The set-up of this model is based on a segmented approach, where the weights of the solid components and the pressures of the liquids (i.e. the permeant and HDL) are calculated over small integral distances along the axis of the permeameter, pertaining to the rotational speed of the centrifuge. The input variables for calculating the stress distributions are detailed in Table 1.

The output values allow a visual assessment of the stress conditions applied to the sample, and graphical outputs take the following forms

Table 1. Input variables for calculating stresses

\begin{tabular}{l|c|c}
\hline Variable description & Units & $\begin{array}{c}\text { Label in } \\
\text { Fig. 5 }\end{array}$ \\
\hline Rotational speed of centrifuge & $\mathrm{r} / \mathrm{min}$ \\
$\mathrm{mm}$ & $\omega$ \\
Length of sample & $\mathrm{mm}$ & $y_{\mathrm{i}}$ \\
Inlet chamber permeant level & $\mathrm{mm}$ & $y_{\mathrm{o}}$ \\
Outlet chamber permeant level & $\mathrm{mm}$ & $r_{\mathrm{b}}$ \\
Radius to bottom of sample & $\mathrm{mm}$ & $R$ \\
Radius to bottom of chamber & $\mathrm{g} / \mathrm{cm}^{3}$ & - \\
Density of the HDL & $\mathrm{mm}$ & - \\
HDL level (distance below the top of the top cap) & $\mathrm{g} / \mathrm{cm}^{3}$ & - \\
Soil bulk density & $\mathrm{g} / \mathrm{cm}^{3}$ & - \\
Density of the permeant &
\end{tabular}

Details of top cap:

Diameter of top cap

Total length of top cap

Thickness of top cap extension wall

Length of top cap extension wall

Thickness of porous disc

Diameter of porous disc

Density of top cap material (stainless steel)

Density of porous stone

\begin{tabular}{c|c}
$\mathrm{mm}$ & - \\
$\mathrm{mm}$ & - \\
$\mathrm{mm}$ & - \\
$\mathrm{mm}$ & - \\
$\mathrm{mm}$ & - \\
$\mathrm{mm}$ & - \\
$\mathrm{g} / \mathrm{cm}^{3}$ & - \\
$\mathrm{g} / \mathrm{cm}^{3}$ & -
\end{tabular}


(a) a comparative assessment of vertical stress in the sample (imposed by the top cap weight and sample weight) and horizontal stress in the sample (imposed by the pressure of the HDL) along the length of the sample

(b) effective confining pressure across the sample (lateral pressure created by the HDL minus the estimated pore water pressure ascertained from permeant pressures/ levels at the inlet (bottom) and outlet (top)).

A trial-and-error approach allows the user to ascertain the appropriate testing variables to use during test set-up to ensure that $(a)$ the sample does not experience large differences in applied lateral and axial stresses; and $(b)$ effective confining pressures are sufficient (given the setting of the gravity spring valve) to negate preferential flow conditions.

As previously detailed, interpretation of permeability values from the results of a centrifuge analysis, neglecting the changes in gravitational acceleration with distance from the point of rotation, can lead to errors in the output values. As such, the energy gradient method of calculating permeability flow (shown in equation (7)), taking into account the changing acceleration with distance from the axis of rotation, has been derived and input into a spreadsheet to ascertain directly the results gained from the centrifuge permeameter designed as part of this research investigation. A derivation is given below based on the methodology proposed by Madabhushi \& Thusyanthan (2003), and the reader should make reference to Fig. 5 for the symbols used in the derived equations.
As previously discussed, a relationship between Darcy's coefficient of permeability and energy gradient per unit volume of fluid may be defined as shown in equation (7).

$$
v=\frac{k_{1 g}}{\gamma_{1 g}} \times \frac{\Delta(u+z \boldsymbol{g} \rho)}{L}
$$

In order to establish an expression for pressure, $u$, consideration must be given to the effects of radius on the gravity. At any point below the water table at radius $r$ from the point of rotation, water pressure may be defined as

$$
u(r)=\int \rho \omega^{2} r d r=\frac{\rho \omega^{2} r^{2}}{2}+C_{1}
$$

where $\rho$ is the density of the permeant used, $\omega$ is the angular velocity of the centrifuge and $C_{1}$ is a constant of integration established by considering the boundary condition of zero pressure at the water table established from permeant level, $y$, inside the inlet or outlet chambers. Fig. 5 shows that at the water table the radius, $r$, equates to $R-y$ (where $R$ is the radius to bottom of inlet and outlet chambers), therefore

$$
C_{1}=-\frac{\rho \omega^{2}}{2}(R-y)^{2}
$$

Substitution of equation (9) into equation (8) establishes an expression for pore-water pressure at any point along the

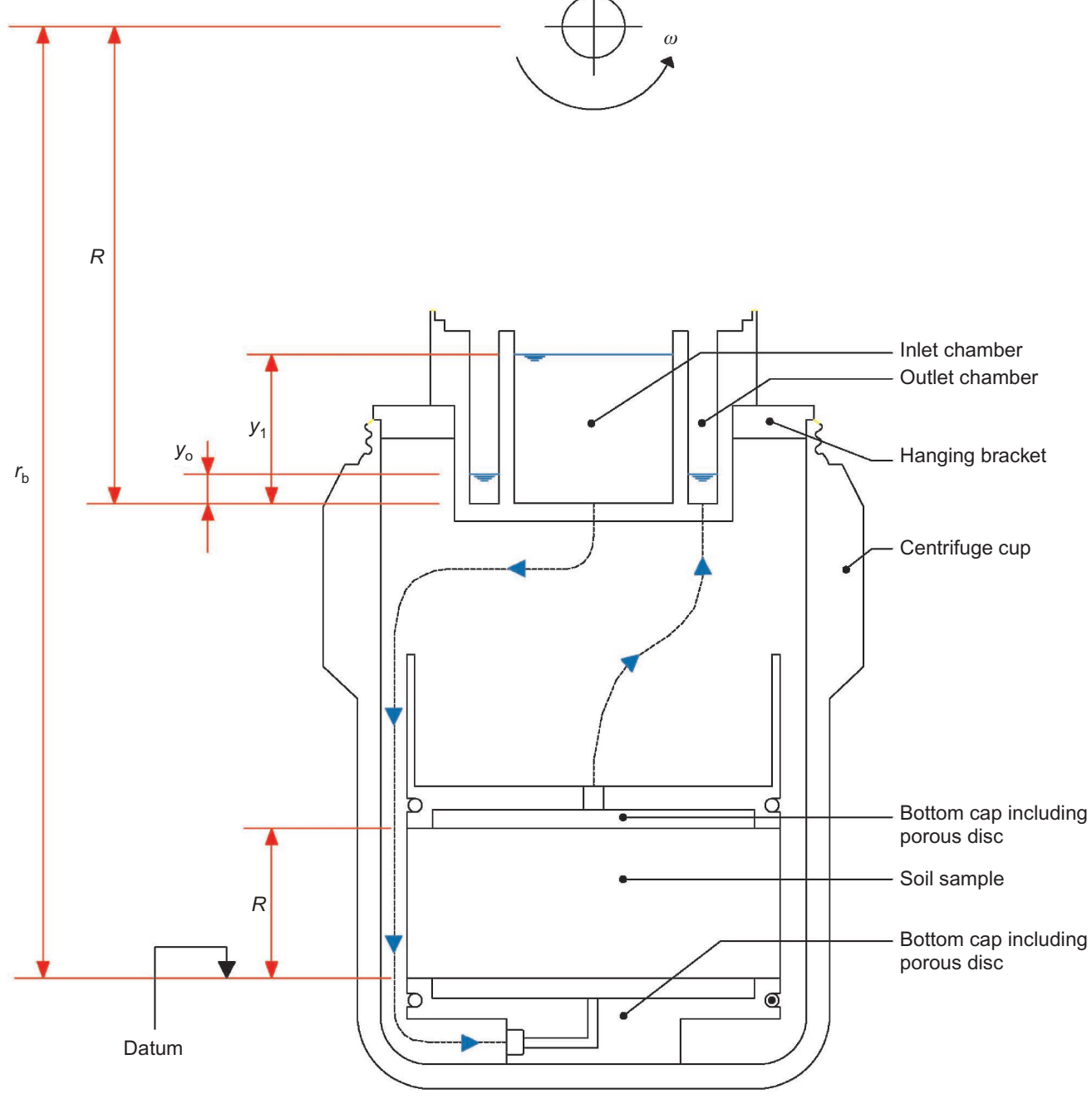

Fig. 5. Centrifuge parameters 
permeameter at radius, $r$, dependent upon the permeant level $y$ inside the inlet or outlet chamber.

$$
u(r)=\frac{\sigma \omega^{2}}{2}\left[r^{2}-(R-y)^{2}\right]
$$

At the base of the sample $r=r_{\mathrm{b}}$ and $y=y_{\mathrm{i}}$, therefore

$$
u_{\mathrm{bot}}=\frac{\sigma \omega^{2}}{2}\left[r_{\mathrm{b}}^{2}-\left(R-y_{\mathrm{i}}\right)^{2}\right]
$$

Similarly, at the top of the sample $r=r_{\mathrm{b}}-L$ and $y=y_{\mathrm{o}}$, therefore

$$
u_{\mathrm{top}}=\frac{\rho \omega^{2}}{2}\left[\left(r_{\mathrm{b}}-L\right)^{2}-\left(R-y_{\mathrm{o}}\right)^{2}\right]
$$

Because of the linear change in gravitational acceleration, it is not correct to assume that the elevation head component at the top of the sample is simply equal to the length of the sample $L$ (i.e. the distance from the datum). Instead an expression representing potential energy $P$ at any distance $d$ from the datum is required. As such, the potential energy may be defined as

$$
\begin{aligned}
P(d) & =z \rho \boldsymbol{g}=\int \rho \omega^{2}\left(r_{\mathrm{b}}-d\right) d d \\
& =\rho \omega^{2}\left(r_{\mathrm{b}} d-\frac{d^{2}}{2}\right)+C_{2}
\end{aligned}
$$

where $C_{2}$ is a constant of integration found by considering the boundary condition $d=0$ when $P=0$. Therefore $C_{2}=0$ and

$$
P(d)=\rho \omega^{2}\left(r_{\mathrm{b}} d-\frac{d^{2}}{2}\right)
$$

At the top of the sample $d=L$ and at the bottom of the sample $d=0$, therefore

$$
P_{\text {top }}=\rho \omega^{2}\left(r_{\mathrm{b}} L-\frac{L^{2}}{2}\right)
$$

and

$$
P_{\text {bot }}=0
$$

From equations (7), (11) and (12), the energy gradient per unit volume of flow may be expressed as

$$
\begin{aligned}
\frac{\Delta(u+z \rho \boldsymbol{g})}{L}= & \frac{\Delta\left(u_{\mathrm{bot}}-\left(u_{\mathrm{top}}+z \rho \boldsymbol{g}_{\mathrm{top}}\right)\right)}{L} \\
& \omega^{2} \Delta\left\{\frac{\rho_{\mathrm{w}}}{2}\left[r_{\mathrm{b}}^{2}-\left(R-y_{i}\right)^{2}\right]\right. \\
& -\frac{\rho_{\mathrm{w}}}{2}\left[\left(r_{\mathrm{b}}-L\right)^{2}-\left(R-y_{\mathrm{o}}\right)^{2}\right] \\
= & \frac{\left.-\rho_{\mathrm{w}}\left(r_{\mathrm{b}} L-\frac{L^{2}}{2}\right)\right\}}{L}
\end{aligned}
$$

Considering equations (7) and (17), an expression for determining permeability using the centrifuge permeability system designed as part of this research may be defined as

$$
\begin{aligned}
k_{1 g}=\frac{\Delta Q \gamma_{1 g}}{A t} \times & \frac{L}{\omega^{2} \Delta\left\{\frac{\rho_{\mathrm{w}}}{2}\left[r_{\mathrm{b}}^{2}-\left(R-y_{\mathrm{i}}\right)^{2}\right]\right.} \\
& -\frac{\rho_{\mathrm{w}}}{2}\left[\left(r_{\mathrm{b}}-L\right)^{2}-\left(R-y_{\mathrm{o}}\right)^{2}\right] \\
& \left.-\rho_{\mathrm{w}}\left(r_{\mathrm{b}} L-\frac{L^{2}}{2}\right)\right\}
\end{aligned}
$$

where $k_{1 g}$ is Darcy's coefficient of permeability of the sample at normal gravitational acceleration, $Q$ is the discharge in a specified time $t$ (i.e. the time between permeant level readings in the inlet and outlet chambers, $y_{i}$ and $y_{0}$ respectively), $A$ is the cross-sectional area of the sample, $\gamma_{1 g}$ is the unit weight of the permeant under normal gravitational acceleration, $\omega$ is the rotational speed of the centrifuge, $L$ is the length of the sample, $\rho_{\mathrm{w}}$ is the density of the permeant, $r_{\mathrm{b}}$ is the radial distance between point of rotation and the bottom of the sample, and $R$ is the radial distance to the base of the inlet and outlet chambers. The relevant units for each of the parameters are listed in Table 2.

As discussed earlier, the values of permeant level in the inlet chamber $y_{i}$ and outlet chamber $y_{0}$ can be accurately correlated with data from the pressure cells. Knowing the cross-sectional area of both the inlet chamber $a_{\mathrm{i}}$ and outlet chamber $a_{\mathrm{o}}$ it is possible to calculate the flow into and out of the sample between readings, that is for a given time interval set by the software controlling the electronics component. Equation (18) can then be used, together with the appropriate calibration factors for the test cell, to determine the coefficient of permeability.

\section{RESULTS AND DISCUSSION OF CENTRIFUGE PERMEABILITY TESTING}

The above formulations allowed the assessment of porewater pressure, vertical pressure and horizontal pressure distributions on the sample during centrifuge testing, along with the parameters listed in Table 2. Fig. 6(a) shows the pore-water pressure variation in the inlet tube (through

Table 2. Centrifuge parameters

\begin{tabular}{l|c|c|c}
\hline Variable & Notation & Magnitude & Unit \\
\hline Radius to bottom of sample & $r_{\mathrm{b}}$ & $198 \cdot 5$ & $\mathrm{~mm}$ \\
Radius to bottom of inlet and chambers & $R$ & 103 & $\mathrm{~mm}$ \\
Length of sample & $L$ & 30 & $\mathrm{~mm}$ \\
Cross-sectional area of inlet chamber & $a_{\mathrm{i}}$ & $803 \cdot 84$ & $\mathrm{~mm}^{2}$ \\
Cross-sectional area of outlet chamber & $a_{\mathrm{o}}$ & $828 \cdot 96$ & $\mathrm{~mm}^{2}$ \\
Cross-sectional area of sample & $A$ & 4415 & $\mathrm{~mm}^{2}$ \\
Time between readings & $\mathrm{d} t$ & 120 & $\mathrm{~min}$ \\
Rotational speed of centrifuge & $\omega$ & 914 & $\mathrm{rev} / \mathrm{min}$ \\
Density of permeant & $\rho_{\mathrm{w}}$ & 1 & $\mathrm{~g} / \mathrm{cm}^{3}$ \\
\hline
\end{tabular}




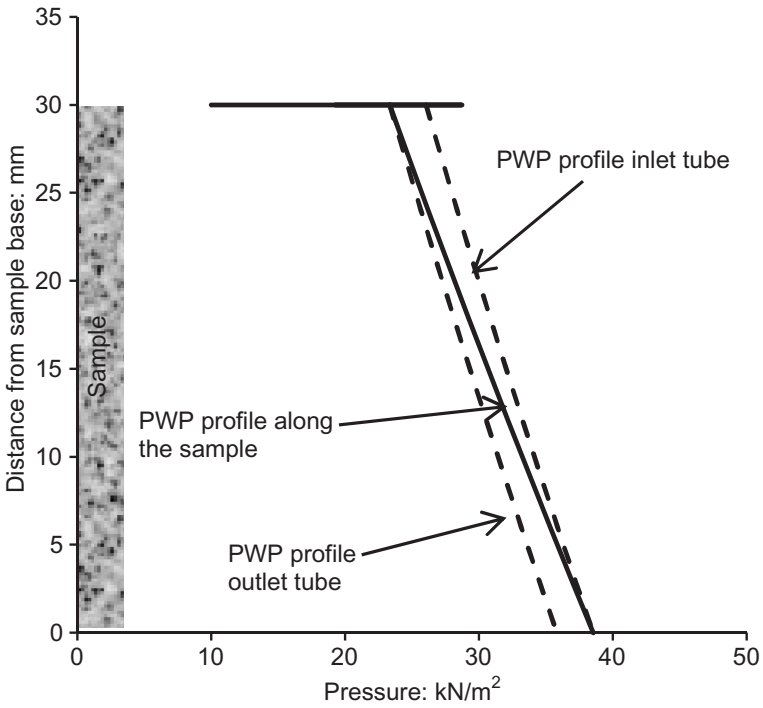

(a)

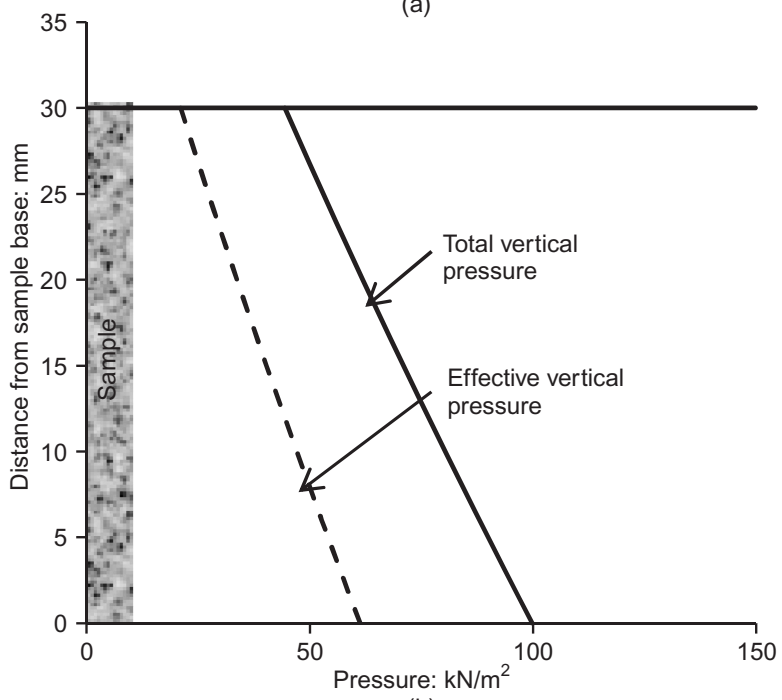

(b)

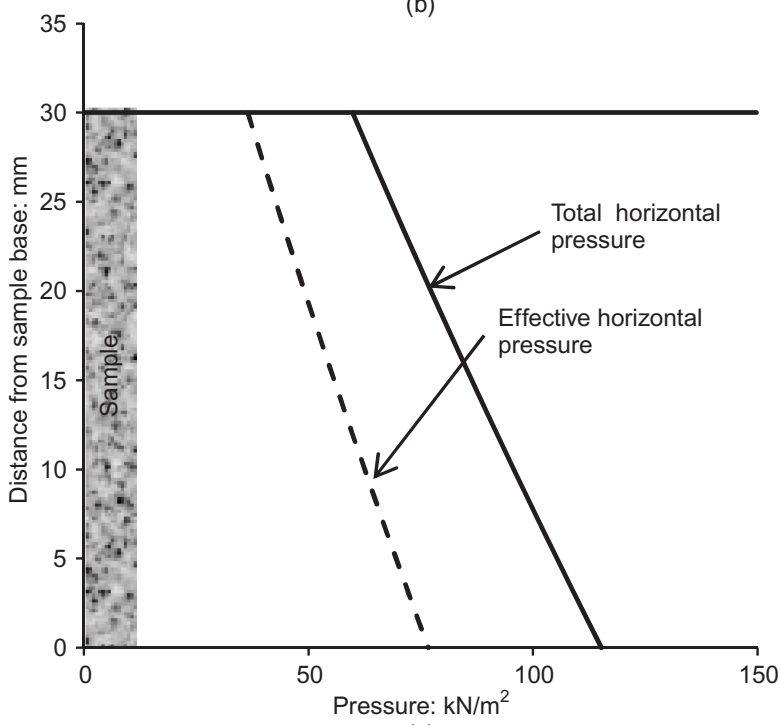

(c)

Fig. 6. (a) Pore water, (b) vertical and (c) horizontal pressures acting on the sample

the base of a $30 \mathrm{~mm}$ high sample), along the length of the sample and in the outlet tube connecting to the outlet chamber. The pore-water pressure was about $38 \mathrm{kPa}$ at the base of the sample and $24 \mathrm{kPa}$ at the top of the sample under gravitation of $250 \mathrm{~g}$ while the inlet chamber was filled with water to its highest level, the outlet being nearly empty. The total, effective vertical and horizontal pressure distributions are shown in Figs 6(b) and 6(c), respectively. The effective lateral pressure is about $38 \mathrm{kPa}$ and it is sufficient to prevent any preferential flow. Analysis was also carried out to predict the inflow/outflow pattern for a given permeability of $7 \cdot 1 \times 10^{-11} \mathrm{~m} / \mathrm{s}$ at an elevated gravitational acceleration equivalent to $250 \mathrm{~g}$. The predicted inflow/outflow patterns using equation (18) are shown in Figure 7, which shows the falling head in the inlet chamber and rising head in the outlet chamber and the required time for stabilisations.

The new device may be used to measure permeability of fine soil; however, its application to highly permeable soils such as sand is limited. For example, for a sample with a relatively high coefficient of permeability of $1 \times 10^{-6} \mathrm{~m} / \mathrm{s}$, the majority of the flow would take place in approximately $1 \mathrm{~min}$ at $100 \mathrm{~g}$. This time frame would allow insufficient data acquisition to accurately determine the coefficient of permeability. However, giving consideration to the typical range of coefficient of permeability values attained for a finegrained sample, say $5 \times 10^{-8} \mathrm{~m} / \mathrm{s}$ to $5 \times 10^{-11} \mathrm{~m} / \mathrm{s}$, the majority of the flow would take place within approximately $38 \mathrm{~min}$ at $100 \mathrm{~g}$ and 7.5 days at $350 \mathrm{~g}$, respectively. These estimates are for the given sample dimensions used in the present research. These estimates indicate that the device developed as part of this research is suitable to measure the permeability of clay liners, where the desired permeability values are typically less than $1.0 \times 10^{-9} \mathrm{~m} / \mathrm{s}(\mathrm{HMG}, 2003)$.

Over the course of the research period, numerous permeability tests were carried out to assess the functionality of the centrifuge permeameter and make adjustments where required. Four permeability tests were carried out using the centrifuge permeameter at an acceleration of $250 \mathrm{~g}$, on reconstituted samples of kaolin clay, and compared with the results from the BS test.

Two sample types were produced by consolidating kaolin slurry of $90 \%$ water content to pressures of $250 \mathrm{kPa}$ and $500 \mathrm{kPa}$ for a period of $72 \mathrm{~h}$, in a one-dimensional consolidation chamber, to produce samples $150 \mathrm{~mm}$ high by $100 \mathrm{~mm}$ in diameter. In total, three sub-samples (one for BS tests and two for the centrifuge permeameter tests) were prepared from the consolidated kaolin, using a sample cutter

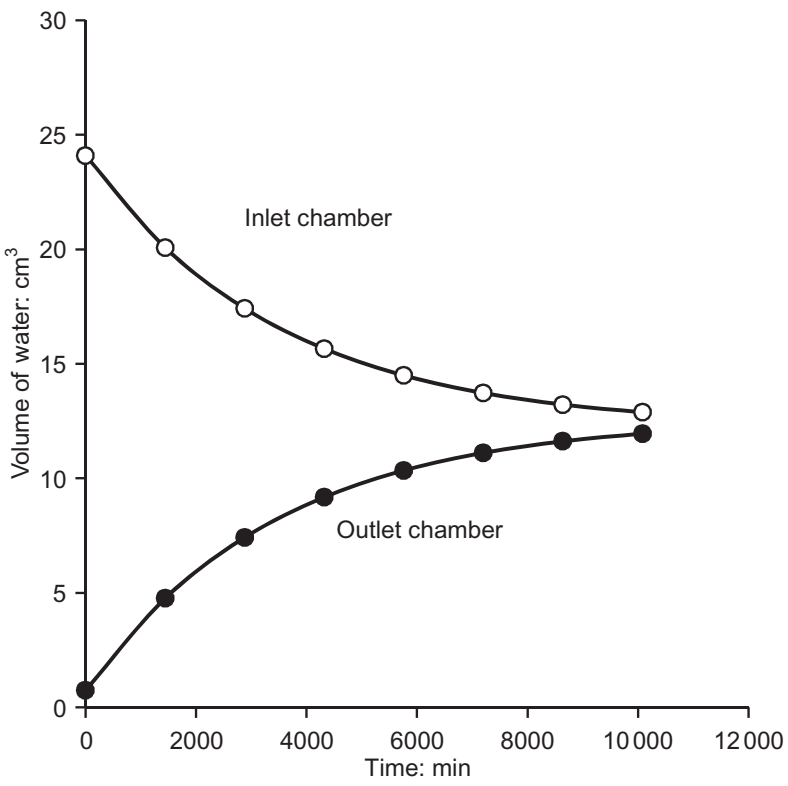

Fig. 7. Predicted inflow and outflow under $250 \mathrm{~g}$ in centrifuge 
of $75 \mathrm{~mm}$ internal diameter, and trimmed to a height of $30 \mathrm{~mm}$. The two BS tests were carried out using a standard permeability cell, following the procedure described in BS 1377: part 6:1990 (BSI, 1990), method 6. The confining pressure, the inlet and outlet pressures were 75, 33 and $25 \mathrm{kPa}$, yielding an effective pressure of $41 \mathrm{kPa}$ with a hydraulic gradient of $26 \cdot 7$, producing an upward flow through the sample. These pressures correspond to average confining pressure, inlet pressure and outlet pressure that the sample experienced in the centrifuge. These BS tests lasted approximately 5 days.

Typical observations in relation to the flow of water from the inlet chamber to outlet chamber in the centrifuge permeameter testing are shown in Fig. 8. Fig. 8(a) shows the inflow/outflow pattern for a sample previously consolidated to $500 \mathrm{kPa}$ and the pattern for a sample consolidated to $250 \mathrm{kPa}$ is shown in Fig. 8(b). The volumes of water in the inflow and outflow chambers were approaching similar values in about 3 days in the case of the sample consolidated to $500 \mathrm{kPa}$ and just over 0.5 days for the sample previously consolidated to $250 \mathrm{kPa}$, although longer time may be needed to achieve complete equalisation of volume of water in the

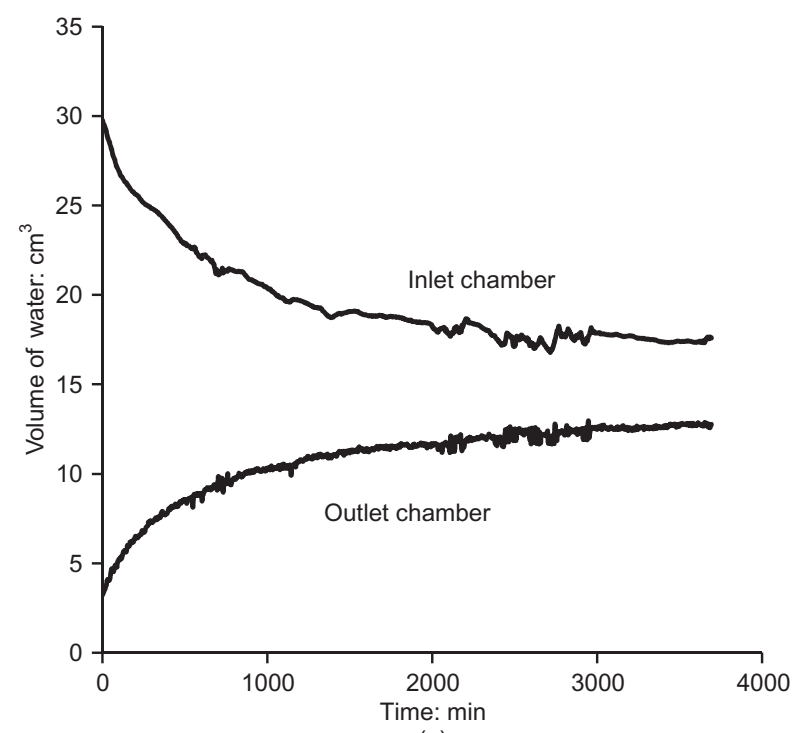

(a)

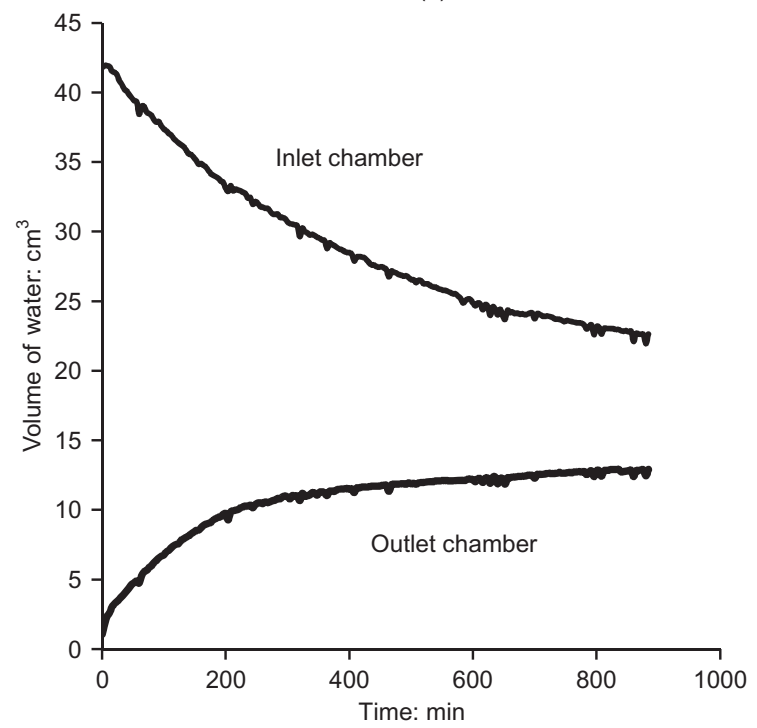

(b)

Fig. 8. Measured inflow-outflow under acceleration of 250g: (a) consolidation pressure $500 \mathrm{kPa}$; (b) consolidation pressure $250 \mathrm{kPa}$ inlet and outlet chambers. In both cases the acceleration was maintained at $250 \mathrm{~g}$. The coefficient of permeability was calculated using equation (18) on an incremental basis. Since the samples were previously consolidated to higher pressure than the pressures applied in the centrifuge (Fig. 6), some elastic swelling was expected, contributing to the difference between the volume of water that flowed into the sample and that collected in the outlet chamber. Once the sample has responded to the stress regime in the centrifuge, the void ratio should remain unchanged and therefore the permeability should be unaffected, except where any an influence due to the change in hydraulic gradient as the head of the inflow is reducing and that for the out flow is increasing. The flow through the sample will stop when the permeant levels in the inflow and outflow chambers equalise. Testing indicates that, compared with the BS tests, a significant saving in test duration, on the order of 1.5-4-fold can be achieved using the centrifuge permeameter. The calculated permeability values using the BS test and the centrifuge permeameter tests are listed in Table 3. The permeability results from the two tests show good correlation, with factors of divergence in test method on analogous samples ranging from 1.01 to $1 \cdot 13$. The results indicate repeatability in the values derived from the centrifuge permeameter apparatus.

As a result, based on these tests it is suggested that the centrifuge permeameter, and its method of analysis, produce acceptable and repeatable results on samples of reconstituted fine-grained material. However, it should be noted that the results obtained from the centrifuge permeameter are limited in number to date; therefore, the conclusions made regarding its abilities need to be rigorously scrutinised by further testing before firm conclusions may be drawn on its viability as an alternative short-duration method of permeability testing for wider application, that is, when testing compacted clays which are initially unsaturated. It is expected that samples which are not fully saturated may pose a problem, in terms of volume of water available in the reservoir to flush the air out of the sample and saturate it. It is possible with the present system for the test to be halted and, if required, the reservoirs can be re-filled; alternatively, a more advanced centrifuge facility could be developed that incorporates a hydraulic rotary union to enable in-flight delivery of fluid.

\section{CONCLUSIONS}

An apparatus was designed and produced to measure water flow through soil specimens under elevated gravity in a small desk-top laboratory centrifuge. The lateral boundary conditions were flexible and tests were carried out using the falling head test method. An inbuilt data acquisition system allowed monitoring of inlet and outlet flow during centrifuge action. The data collected were analysed using a mathematical model, which is presented in the paper. The following conclusions are drawn.

Table 3. Calculated permeability using BS and centrifuge permeameter

\begin{tabular}{l|c|c|c}
\hline $\begin{array}{l}\text { Initial consolidation } \\
\text { pressure: } \mathrm{kPa}\end{array}$ & Test type & $\begin{array}{c}\text { Initial void } \\
\text { ratio }\end{array}$ & $\begin{array}{c}\text { Calculated } \\
\text { permeability: m/s }\end{array}$ \\
\hline 250 & $\mathrm{CP}^{*}$ & 1.425 & $4.32 \times 10^{-10}$ \\
250 & $\mathrm{CP}$ & 1.432 & $4.44 \times 10^{-10}$ \\
250 & $\mathrm{BS}$ & 1.423 & $4.63 \times 10^{-10}$ \\
500 & $\mathrm{CP}$ & 1.361 & $9.80 \times 10^{-11}$ \\
500 & $\mathrm{CP}$ & 1.365 & $1.12 \times 10^{-10}$ \\
500 & BS & 1.361 & $9.89 \times 10^{-11}$ \\
\hline
\end{tabular}

* $\mathrm{CP}$, centrifuge permeameter. 
(a) The calculated permeability of reconstituted kaolin, consolidated to different pressures using the centrifuge permeability device, agrees well with the permeability values calculated using the data generated from the BS test.

(b) The duration of testing in the centrifuge is relatively short. However, it should be noted that a complete equilibrium of water in inlet and outlet chambers may require more time, although that is not essential for determining the permeability value.

(c) Further investigation is required to assess permeability of compacted soils, in which the duration of the testing can be expected to be higher due to the unsaturated nature of the soil at the start of testing.

\section{ACKNOWLEDGEMENTS}

The authors would like to thank Mr Kenny McDonald (chief technician, electronic laboratory, SPACE, Queen's University Belfast) and Mr Jim Knox (head of technicians, CNC workshop, Faculty of Engineering, Queen's University Belfast) for their input into the research.

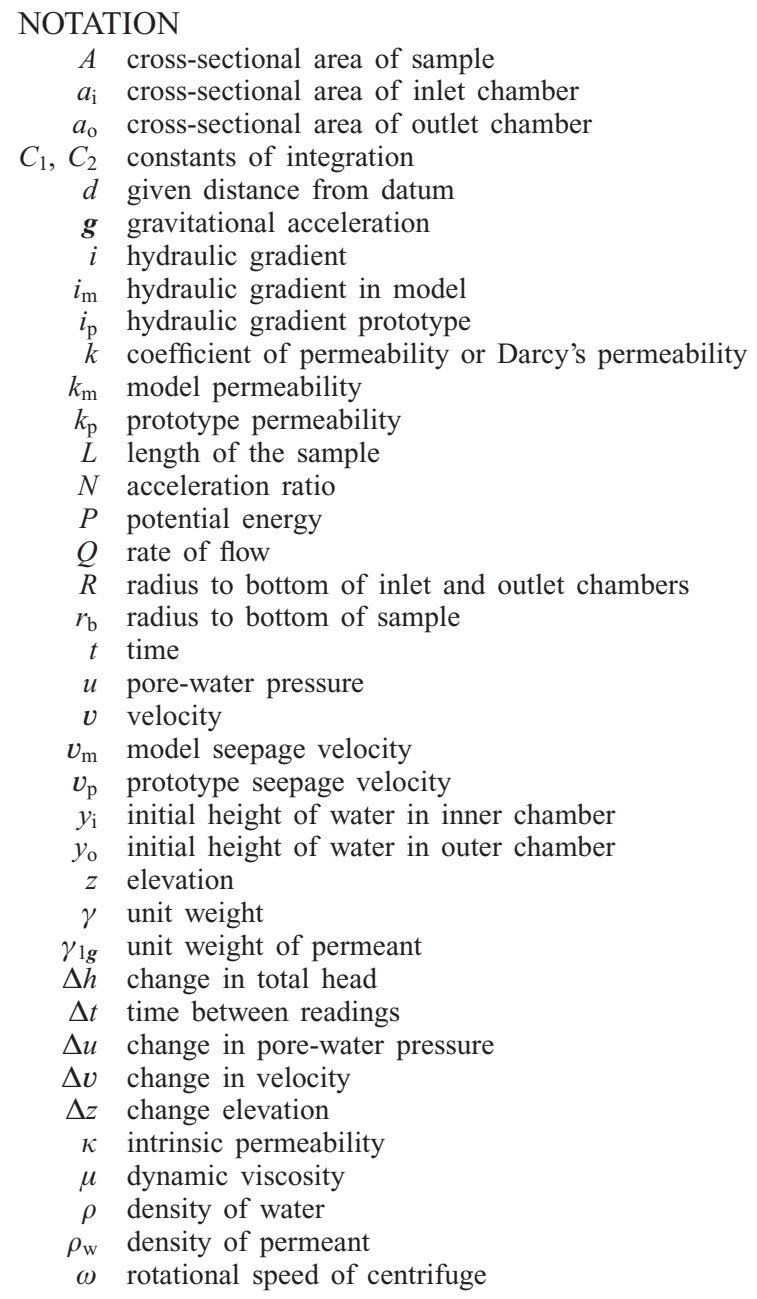

\section{REFERENCES}

Aiban, S. A. \& Znidarcic, D. (1989). Evaluation of the flow pump and constant head techniques for permeability measurements. Géotechnique 39, No. 4, 655-666, http://dx.doi.org/10.1680/ geot.1989.39.4.655.

Arulanandan, K., Thompson, P. Y., Kutter, B. L., Meegoda, N. J., Muraleetharan, K. K. \& Yogachandran, C. (1988). Centrifuge modeling of transport processes for pollutants in soils. J. Geotech. Engng 114, No. 2, 185-205.
Black, J. A. (2014). Development of a small scale teaching centrifuge. In ICPMG2014 - Physical modelling in geotechnics: Proceedings of the 8th international conference on physical modelling in geotechnics (eds C. Gaudin and D. White), pp. 187-192. Boca Raton, FL, USA: CRC Press.

Bolton, M. D. \& Powrie, W. (1988). Behaviour of diaphragm walls in clay prior to collapse. Géotechnique 38, No. 2, 167-189, http://dx.doi.org/10.1680/geot.1988.38.2.167.

Brown, J. L., Sivakumar, V., McKinley, J. D., Harmon, N. \& McDonald, K. (2009). The miniature wireless data-logger for pressure measurements in geotechnical applications. Géotechnique 59, No. 2, 141-149, http://dx.doi.org/10.1680/geot.2007.00133.

BSI (1990). BS 1377:1990: Methods of tests for soils for civil engineering purposes, code of practice. London, UK: BSI.

Bucky, P. B. (1931). The use of models for the study of mining problems, Technical Publication 425. New York, NY, USA: American Institute of Mining and Metallurgical Engineers.

Butterfield, R. (2000). Scale-modelling of fluid flow in geotechnical centrifuges. Soils Found. 40, No. 6, 39-45.

Cargill, K. W. \& Ko, H. Y. (1983). Centrifugal modeling of transient water flow. J. Geotech. Engng 109, No. 4, 536-555.

Dean, E. T. R. (2001). Discussion on 'Scale-modelling of fluid flow in geotechnical centrifuges'. Soils Found. 41, No. 4, 108 110.

Environment Agency (2003). Validation of the accelerated permeability test for landfill engineering, R\&D Technical Summary P1-398/TS. Hatfield, UK: Environment Agency.

Garnier, J., Gaudin, C., Springman, S. M., Culligan, P. J., Goodings, D. J., Konig, D., Kutter, B. L., Phillips, R., Randolph, M. F. \& Thorel, L. (2007). Catalogue of scaling laws and similitude questions in geotechnical centrifuge modelling. Int. J. Phys. Modelling Geotech. 7, No. 3, 1-23.

Goodings, D. J. (1979). Centrifugal modelling of soil structures. Part I. Centrifugal modelling of slope failures. $\mathrm{PhD}$ thesis, University of Cambridge, UK.

Goodings, D. J. (1985). Relationships for modelling water effects in geotechnical centrifuge models. In Application of centrifuge modelling to geotechnical design: Proceedings of a symposium on the application of centrifuge modelling to geotechnical design, Manchester, 16-18 April 1984 (ed. W. Craig). Rotterdam, the Netherlands: Balkema.

HMG (Her Majesty's Government) (2003). Environmental protection. The landfill regulations (Northern Ireland), Statutory Rules of Northern Ireland, 2003 no. 496. London, UK: The Stationery Office.

Huang, S., Barbour, S. L. \& Fredlund, D. G. (1998a). Development and verification of a coefficient of permeability function for a deformable unsaturated soil. Can. Geotech. J. 35, No. 3, 411425.

Huang, S., Fredlund, D. G. \& Barbour, S. L. (1998b). Measurement of the coefficient of permeability for a deformable unsaturated soil using a triaxial permeameter. Can. Geotech. J. 35, No. 3, 426-432.

Khalifa, A., Garnier, J., Thomas, P. \& Rault, G. (2000). Scaling laws of water flow in centrifuge models. In Proceedings of the international symposium on physical modelling and testing in environmental geotechnics, La Baule, France (eds J. Garnier, L. Thorel and E. Haza), pp. 207-216. Paris, France: Laboratoire Central des Ponts et Chaussées.

Lambe, T. W. \& Whitman, R. V. (1979). Soil mechanics (SI version). New York, NY, USA: Wiley.

Madabhushi, S. P. G. \& Thusyanthan, N. I. (2003). Scaling of seepage flow velocity in centrifuge models. Cambridge, UK: Cambridge University Press.

Mitchell, J. K. (1993). Fundamentals of soil behaviour, 2nd edn. New York, NY, USA: Wiley.

Mitchell, R. J. (1994a). Centrifuge techniques for testing clay liner samples. Can. Geotech. J. 31, No. 4, 577-582.

Mitchell, R. J. (1994b). Flexible, no lateral strain apparatus for clay liner-leachate testing. In Centrifuge '94: Proceedings of the 1994 international conference, Singapore (eds F. H. Lee, C. F. Leung and T. S. Tan), pp. 351-351. Boca Raton, FL, USA: CRC Press.

Mitchell, R. J. (1998). The eleventh annual R. M. Hardy keynote address, 1997: Centrifuge in geoenvironmental practice and education. Can. Geotech. J. 35, No. 4, 630-640. 
Muir-Wood, D. (2004). Geotechnical modelling. London, UK: Spon. Muskat, M. (1937). The flow of fluids through porous media. J. Appl. Phys. 8, No. 4, 274-282.

Olsen, H. W., Nichols, R. W. \& Rice, T. L. (1985). Low gradient permeability measurements in a triaxial system. Géotechnique 35, No. 2, 145-157, http://dx.doi.org/10.1680/geot.1985.35.2.145.

Schofield, A. N. (1980). Cambridge geotechnical centrifuge operations. Géotechnique 30, No. 3, 227-268, http://dx.doi.org/ 10.1680/geot.1980.30.3.227.

Singh, D. N. \& Gupta, A. K. (2000). Modelling hydraulic conductivity in a small centrifuge. Can. Geotech. J.37, No. 5, $1150-1155$.

Singh, D. N. \& Gupta, A. K. (2001). Falling head hydraulic conductivity tests in a geotechnical centrifuge. J. Testing and Evaluation 29, No. 3, 258-263.

Singh, D. N. \& Gupta, A. K. (2002). Modelling hydraulic con- ductivity in a small centrifuge: Reply. Can. Geotech. J. 39, No. 2, 488-489.

Take, W. A. \& Bolton, M. D. (2011). Seasonal ratcheting and softening in clay slopes, leading to first-time failure. Géotechnique 61, No. 9, 757-769, http://dx.doi.org/10.1680/geot.9.P.125.

Tan, T. S. \& Scott, R. F. (1985). Centrifuge scaling considerations for fluid-particle systems. Géotechnique 35, No. 4, 461-470, http://dx.doi.org/10.1680/geot.1985.35.4.461.

Taylor, R. N. (1987). Discussion on Tan \& Scott (1985). Géotechnique 37, No. 1, 131-133, http://dx.doi.org/10.1680/geot.1987.37.1.131.

Theriault, J. A. \& Mitchell, R. J. (1997). Use of a modelling centrifuge for testing clay liner compatibility with permeants. Can. Geotech. J. 34, No. 1, 71-77.

White, D. J. (2008). Contributions to Geotechnique 1948-2008: Physical modelling. Géotechnique 58, No. 5, 413-421, http:// dx.doi.org/10.1680/geot.2008.58.5.413. 